\title{
Energy-efficient Transmission based on Hierarchical Routing Protocol for Wireless Sensor Networks
}

\author{
M. Ben Salah, A. Boulouz \\ Laboratoire des systèmes informatiques et vision LabSIV \\ Faculté des Sciences Université Ibn Zohr, Agadir, Maroc
}

\begin{abstract}
In wireless sensor networks (WSN) energy consumption is the most critical problem because each sensor node has limited batteries. In order to reduce energy consumption and to optimize the network life-time of this kind of networks clustering algorithms have been widely used. LEACH is the most popular clustering algorithm, which used an interesting technique to select a cluster head alternately and to build cluster. In this work, we propose a new scheme to select cluster head according to the residual energy of nodes. The simulation results show that proposed algorithm achieve longer stability period and network life-time by comparison to other clustering approaches i.e. LEACH, ALEACH, LEAHCDCHS and LEACH-SWDN.
\end{abstract}

\section{Keywords}

Wireless sensor networks; energy consumption; clustering; homogeneous; life-time, stability period

\section{INTRODUCTION}

Many advanced work have been done in WSN which is used in many applications as home security, healthcare applications, intrusion detection, sensing ambient conditions such as sound, pressure, gas concentration and temperature [1, 2]. A wireless sensor network has characteristics of selforganization and energy constraints [3, 4].

A WSN consists of a large number of sensor nodes that deployed in an area of interest (general inaccessible areas). All sensor nodes should collaborate with each other in order to transmit their sensed data to the base-station (BS) and finally accomplish the sensing and communication task. In these kinds of network wireless transmission is the most energy consuming operation and sensors nodes are limited battery. Therefore, energy efficiency is a very crucial issue [5-6] and energy consumption transmission protocol is required to maximize network lifetime.

Energy consumption is more studied and modeled to generate protocols and models for improvement of the performance as particularly a lifetime of WSN which is extremely important [5-14]. Clustering algorithms were most used [8-15]. The principle is to divide the network into clusters. For Each cluster a $\mathrm{CH}$ node is designated. The member nodes send data to the $\mathrm{CH}$ that will route them to the $\mathrm{BS}$ after aggregation.

LEACH [8] is the best-known clustering algorithm for wireless sensor network. The good idea of this algorithm has allowed several researchers to do an improvement and even draw inspiration for new algorithm for better control and energy conservation in WSN as the protocols: LEACH-DCHS [9], ALEACH [10], LEACH-SWDN [11]. LEACH is based on weighted election probabilities assigned to each node to become $\mathrm{CH}$ without taking account to their residual energy. Some nodes are more frequently becomes $\mathrm{CHs}$ and spend most of energy. It may happen after some rounds the energy of this nodes becomes less than other nodes and the network becomes heterogeneous on energy. To overcome this drawback, this work presents, a new $\mathrm{CH}$ selection scheme tacking account residual energy of each node and the threshold energy of live nodes. By using these criteria, we hope increases stability and lifetime of network.

\section{LEACH PROTOCOL}

LEACH is the first adaptive hierarchical clustering routing protocol $[8,12]$. The idea of LEACH is to organize WSN into clusters. Each cluster has a $\mathrm{CH}$ whose using as local routers to the BS: Each node transmits sensed data to its closest $\mathrm{CH}$. The $\mathrm{CH}$ for each cluster after receiving all the data from cluster members performs aggregation and then transmits both an aggregated packet to the BS through a single-hop relay in order to reduce the amount of information transmitted to the base station.

LEACH consists of two phases: the set-up phase, and the steady-state phase. In setup phase, a few nodes are selected randomly as cluster heads (CHs) and nodes are organized into clusters and in the steady state, the time slot scheduling is creating and the data transmission to the BS is held.

$\mathrm{CHs}$ has to consume more energy as compare to member nodes, in order to balance the energy of the network LEACH operates in several rounds, each consisting of a set-up and a steady-state phase.

In the set-up phase one node is selected as $\mathrm{CH}$ based on the threshold T(i) given by eq. 1 and the generated random number. The threshold T(i) is defined as follows [8]:

$$
T(i)=\left\{\begin{array}{l}
\frac{p}{1-p^{*}\left(r \bmod \frac{1}{p}\right)} \\
0 \text { otherwise }
\end{array}\right.
$$

Where $p$ is-the cluster head probability, $\mathrm{G}$ is the set of nodes that have not been cluster heads in the last $1 / p$ rounds and $r$ is the current round.

In LEACH each node becomes a cluster head only for a single time for $r=\frac{1}{p}$ rounds. In the beginning of each rounds every node belong to $\mathrm{G}$ generates a uniform random number in the interval $[0,1]$. The node is selected as a cluster head for the current round if the random number is less than the $\mathrm{T}(\mathrm{i})$ otherwise the node is expected to join the nearest cluster head in its neighborhood. 


\section{THE PROPOSED MODIFIED LEACH PROTOCOL}

LEACH randomly selects a cluster heads and there are no restrictions on their distribution and on their energy level as showing in eq. 1 . This is meaning that nodes with low residual energy may be selected as cluster head with the same probability of those with high residual energy. As mentioned above LEACH let every node to become $\mathrm{CH}$ one in every $\frac{1}{p}$ rounds. With a paste round, it is obvious that all the nodes do not the same residual energy. So, if the period $1 / \mathrm{p}$ which one node can become $\mathrm{CH}$ is kept equal for all nodes as in LEACH the energy is not uniformly distributed and nodes having low energy can be $\mathrm{CH}$ and die quickly before nodes with high energy. Therefore, the level of the residual energy of the nodes should be considered in order to making nodes with higher residual energy level have the bigger probability to elect itself to be cluster head. In order to take account, the residual energy, the average probability for cluster head must be depending to residual energy and can be given as:

$$
p i=p o \frac{E_{r}(i)}{E o}
$$

The threshold value for each node to become a cluster head during each round becomes:

$$
T(i)=\frac{p i}{1-p i *\left(r \bmod \frac{1}{p i}\right)} \text { if } \mathrm{s} \in \mathrm{G}
$$

Doing this, the nodes with higher energy have more chance to become $\mathrm{CH}$. With this approach, it is more likely to ensure energy balance of the entire network, prolong the network stability period and lifetime.

\subsection{Algorithm Description}

In each round nodes decides to become $\mathrm{CH}$ based upon pi and threshold T(i) given in eq. 2 and eq. 3 respectively. Indeed, each node having residual energy great or equal Ev generates a uniform random number in the interval $[0,1]$, if this number is less than the threshold $\mathrm{T}(\mathrm{s})$, then this node is candidate to become a cluster head. The proposed algorithm comports two phases: set-up phase and steady-state phase as follows:

BEGIN

//Set-up phase :



$\mathrm{CHs}$ broadcast $\mathrm{CH}$-status;

non- $\mathrm{CH}$ send join-request message to

closest- $\mathrm{CH}$

Clusterss(c); //form a cluster c end if

// Steady-state phase: Similar than LEACH

if $($ node $(\mathrm{i})=\mathrm{CH})$ then

Receive data from members;

Aggregate and transmit received data to $\mathrm{BS}$;

else

if (TimeSlot) then

transmit data to associate $\mathrm{CH}$;

else

node $i$ at a sleep state;

end if

end if

END

\section{RADIO DISSIPATION}

The energy required by the transmit amplifier $T_{T x}(l, d)$ to transmit 1 bit message between a transmitter and receiver over a distance $d$ is [8-12]:

$T(i)= \begin{cases}l \times E_{\text {elec }}+l \times \varepsilon_{f s} \times d^{2} & \text { if } \mathrm{d} \leq \mathrm{d}_{0} \\ l \times E_{\text {elecs }}+l \times \varepsilon_{m p} \times d^{4} & \text { if } \mathrm{d} \geq \mathrm{d}_{0}\end{cases}$

Where $\mathrm{d}_{0}=\sqrt{\varepsilon_{f s} / \varepsilon_{m p}}$ is the threshold distance, Eelec represents the energy consumption in the electronics for sending or receiving one bit. The terms $\varepsilon_{f s} \times \mathrm{d}^{2}$ and $\varepsilon_{m p} \times \mathrm{d}^{4}$ represent respectively the amplifier energy consumptions for a short and long-distance transmissions .

\section{SEMULATIONS AND RESULTS}

This section evaluates the performance of proposed protocol using MATLAB. We will interest about network lifetime (N.L.T: time period from the start of the network until the death of the last node), period stability (PS: time period from the start of the network until the death of the first node) and energy consumption.

The Lifetime of a network refers to the time period from the start of the network until the death of the last node.

We consider a WSN with 100-node sensor randomly deployed inside $100 \mathrm{~m} \times 100 \mathrm{~m}$ (Fig. 1). The BS is located at coordinate $(50,175)$.

The simulation parameters used are listed in Table 1 according to the radio basic energy dissipation model [7-10] 


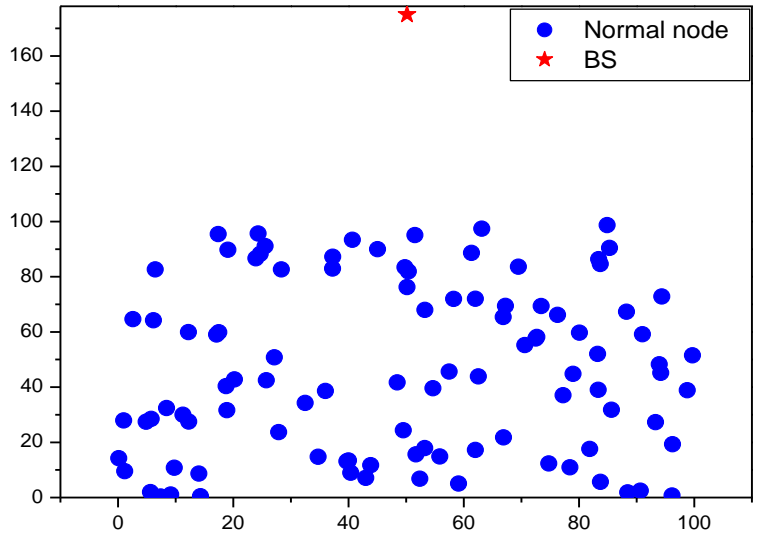

Fig. 1: Random deployment of sensors nodes

(100 nodes, 100m x100m).

Fig. 2 show comparison between the proposed protocol and other clustering approaches i.e. LEACH, ALEACH, LEAHCDCHS and LEACH-SWDN regarding number of a live nodes. It can be seen that the proposed protocol prolonged lifetime network significantly compared with all other protocol. In our case the network remains alive almost up to 3000 rounds assuring network life-time to be more optimized. Moreover, it is also obvious that stability period is also improved i.e., first node dies around 1740 rounds whereas, in schemes like LEACH, ALEACH, LEAHC-DCHS and LEACH-SWDN this value is much lower.

Table 1: Simulation parameters

\begin{tabular}{|c|c|}
\hline Parameters & values \\
\hline Number of nodes & 100 \\
\hline Field simulation & $100 \mathrm{~m} \times 100 \mathrm{~m}$ \\
\hline Base station position & $(50,175)$ \\
\hline Initial energy (E0) & $0.5 \mathrm{~J}$ \\
\hline Transmitter Electronics (Eelec) & $50 \mathrm{~nJ} / \mathrm{bit}$ \\
\hline Receiver Electronics (Eelec) & $50 \mathrm{~nJ} / \mathrm{bit}$ \\
\hline Length of data packet (Bytes) & 3000 \\
\hline $\begin{array}{l}\text { Transmitter Amplifier }\left(\varepsilon_{\mathrm{fs}}\right) \\
\left.\text { (If } \mathrm{d}<\mathrm{d}_{0}\right)\end{array}$ & $10 \mathrm{pJ} / \mathrm{bit} / \mathrm{m}^{2}$ \\
\hline $\begin{array}{l}\text { Transmitter Amplifier }\left(\varepsilon_{\mathrm{mp}}\right) \\
\text { (If } \mathrm{d}>\mathrm{d}_{0} \text { ) }\end{array}$ & $0.0013 \mathrm{pJ} / \mathrm{bit} / \mathrm{m}^{4}$ \\
\hline Probability of $\mathrm{CH}$ & 0.05 \\
\hline
\end{tabular}

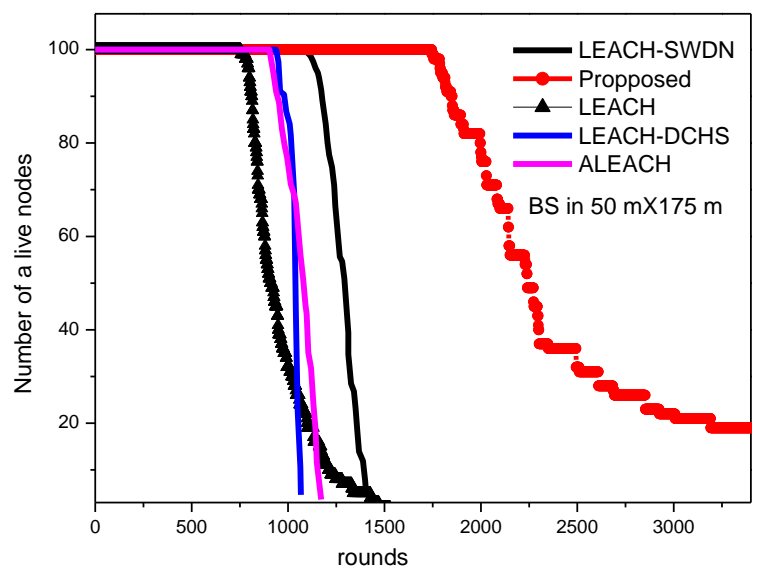

Fig. 2: Wireless Sensor Network lifetime (BS in $50 \mathrm{~m} \times 175 \mathrm{~m})$
Table 2: Comparison of network parameters

\begin{tabular}{|l|l|l|l|l|l|}
\hline & LEACH & ALEACH & $\begin{array}{l}\text { LEAHC- } \\
\text { DCHS }\end{array}$ & $\begin{array}{l}\text { LEACH- } \\
\text { SWDN }\end{array}$ & proposed \\
\hline S.P & 750 & 900 & 950 & 1115 & 1740 \\
\hline N.L.T & 1750 & 1066 & 1172 & 1400 & $>3000$ \\
\hline
\end{tabular}

Table 2 show comparison of network parameters ie. network lifetime (N.L.T) and stability period (S.P). From table 2 it is clear that the proposed protocol performs quiet well comparing with LEACH,ALEACH, LEAHC-DCHS and LEACH-SWDN

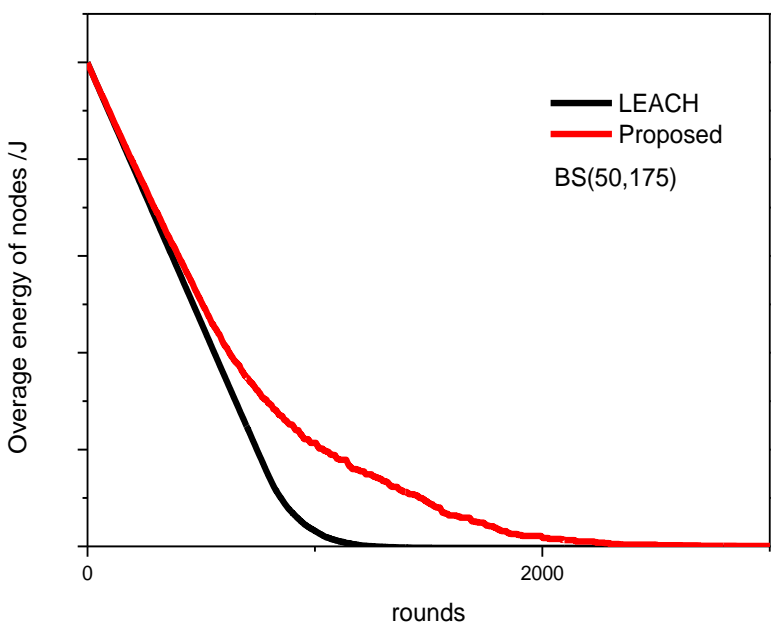

Fig.3 The energy consumption

Fig. 3 compared the energy consumption between the LEACH and our protocol during the simulation time. Dissipation of energy is due to the operations performed, such as sensing operations, data processing and communication. It is shown clearly that our protocol consumes less energy than LEACH.

\section{CONCLUSION}

In this paper, we proposed an algorithm based on LEACH to improve WSN lifetime. In the proposed routing protocol, we consider the residual energy to select $\mathrm{CH}$ nodes. Presented simulation results shows that the improved algorithm can balance the network energy consumption, prolong the network lifetime and increase the stability period. As future work, study of proposed protocol is envisaged, in the case of a network with sensor nodes heterogeneous.

\section{REFERENCES}

[1] Y. He, W. S. Yoon and J. H. Kim. "Multi-levelClustering Architecture for Wireless Sensor Networks". Information Technology Journal, Vol. 5, No. 1, 2006, pp. 188-191

[2] Su Yanjing, He Yanjun, Zhang Beibei, Liu Xue Mining, Science and Technology (China) 21 2011, pp845-850

[3] X. Guan, W. H. Yang and D. G. Bi, "EEHCA: An Energy-Efficient Hierarchical Clustering Algorithm for Wireless Sensor Networks", Information Technology Journal, Vol. 7, No. 2, 2008, pp. 245-252.

[4] J. Yang and D. Zhang, "An Energy-Balancing Unequal Clustering Protocol for Wireless Sensor Networks", Information Technology Journal, Vol. 8, No. 1, 2009, pp. $57-63$ 
[5] C. Alippi, G . Anastasi, MD Francesco, Roveri M, ' An adaptive sampling algorithm for effective energy management in wireless sensor networks with energyhungry sensors", IEEE Trans Instrum Meas 2010;59(2):335-44.

[6] US Sutar, SK Bodhe, ''Energy efficient topology control algorithm for multi-hop ad-hoc wireless sensor network', In: Proc. 3rd IEEE international conference on computer science and information technology (ICCSIT), Chengdu, China; July 2010

[7] Hai-Ying Zhou, Dan-Yan Luo, Yan Gao, De-Cheng Zuo, "'Modeling of Node Energy Consumption for Wireless Sensor Networks", Wireless Sensor Network, 2011,3, $18-23$

[8] W. Heinzelman, A. Chandrakasan, and H. Balakrishnan, "Energy-efficient routing protocols for wireless microsensor networks," in Proc. 33rd Hawaii Int. Conf. System Sciences (HICSS), Maui, HI, Jan. 2000.

[9] Handy MJ, Haase M, Timmermann D. Low energy adaptive clustering hierarchy with deterministic clusterhead selection. In: Proc. 4th IEEE conference on mobile and wireless communication networks; 2002. p. 368-72.

[10] S. Ali Md, D. Tanay, B. Rahul, "ALEACH advanced LEACH routing protocol for wireless microsensor networks", In: Proc. ICECE 2008, vols. 1 and 2; 2008. p. 909-14.

[11] A.Wang, D. Yang, D. Sun, “A clustering algorithm based on energy information and cluster heads expectation for wireless sensor networks". Computers and Electrical Engineering 38 (2012) 662-671

[12] W.R.Heinzelman, A.P.Chandrakasan, H.Balakrishnan, "An application- specific protocol architecture for wireless microsensor networks", IEEETransactions on Wireless Communications (4) (2002) 660-670.

[13] F. Comeaua, N. Aslamb, " Analysis of LEACH Energy Parameters", Workshop on Emerging Topics in Sensor Networks (EmSeNs 2011), Procedia Computer Science 5 (2011) 933-938

[14] Lin Xiao,1,2 Fahui Wu,1 Dingcheng Yang,1 Tiankui Zhang, Xiaoya Zhu, "Energy Efficient Wireless Sensor Network Modelling Based on Complex Networks " Journal of Sensors, 2016 (2016).

[15] Rohit D. Gawade and S. L. Nalbalwar," A Centralized Energy Efficient Distance Based Routing Protocol for Wireless Sensor Networks", Journal of Sensors, 2016 (2016). 